\title{
QUALITY IMPROVEMENT OF DAMIETTA CHEESE USING SOME SPICES EXTRACT
}

\author{
IBRAHIM, JEHAN I. and ABDEL-HAKIEM E H.
}

Department of Food Hygiene and Control, Faculty of Vet. Med., Sues canal University, Ismailia, Egypt.

Email: emamhu@yahoo.com.

Assiut University web-site: www.aun.edu.eg

Received at: 19/8/2015

Accepted: 2/9/2015

\section{ABSTRACT}

The experiment was carried out to investigate the effect of some spices extract on the quality of Damietta cheese. The sensory evaluation of cheese supplemented with cumin oil only gave slightly pronounced flavor, compared with control one. The curcumin treated cheese showed faint-yellow color of slight good appearance. The microbiological quality revealed that cumin and N.sativa oils have strong antimicrobial effect against microbial groups than their aqueous extracts. Curcumin was found to be the most effective additives among the aqueous spices extract used. According to the effect of storage periods, there was a significant reduction of microbial groups in treated cheeses. The multiplication rates of microbial groups in control cheeses of $5 \%$ salt were rapid compared with control one of $10 \%$ salt during the storage periods. On contrary, the reduction rates of microbial groups were rapid in treated cheeses of $10 \%$ salt compared with $5 \%$ salt. Also, there was a high significant difference between treated cheeses and control one. Concerning salt concentration, there was a significant difference between both types of cheese $(5 \&$ $10 \%$ salt) against all microbial groups, except S.aureus. Also, there was a significant difference for all microbial groups between cheeses of $5 \%$ and $10 \%$ salt in all cheese groups, except S.aureus. Fungal growth was observed at 21 days of storage over cheese surface and whey of control one of $5 \%$ salt only.

Key words: Spices extract, Damietta cheese.

\section{INTRODUCTION}

Cheese is a food derived from different types of milk that is produced in a wide range of flavors and textures. There are several types of cheese which are grouped according to different criteria such as length of ageing, texture, manufacturing method, fat content, animal milk, country, etc. Damietta and Kareish cheeses are the most popular soft cheese in Egypt, usually made from salted raw whole or skim milk that are previously heated at $40^{\circ} \mathrm{C}$ by using rennet extract. Cheese made from raw milk usually carries large number of pathogenic or non-pathogenic microorganisms. In addition, cheese may be subjected to contamination during processing, handling and distribution from different sources by various types of microorganisms (Yousef et al., 2001). Different outbreaks were reported as a result of consumption of different types of cheese (WHO, 2002).
Common herbs or spices may be added to some cheeses as flavoring agents including N.sativa, bitter cumin, red and green peppers, garlic, black pepper, caraway, parsley (Hassanein et al., 2014). Levels of the additives are typically less than $1 \%$ of the curd (Hayaloglu and Farkye, 2011). Spices as natural plant foodstuffs are common dietary adjuncts that contribute to the taste and flavor of food, stabilize the food from microbial deterioration and offer a promising for food safety. It was recorded that spices and their derivatives have inhibitory activity on the growth of bacteria, fungi and their toxins, so, they could be used in food industry to assure the production of microbiologically stable food (Kizil and Sogut, 2003). Therefore, this study was carried out to investigate the effect of some spices extract on sensory attributes and microbiological quality of Damietta cheese supplemented with these spices. 


\section{MATERIALS and METHODS}

\section{Preparation of spices extract}

Bitter Cumin and N.sativa aqueous extracts were prepared according to (Arora and Kaur 1999), where good quality seeds were first cleaned using sterile dist. water to remove any dirt or debris, dried in laminar flow biological safety cabinet, and then ground using sterile electric blender. Water extract was prepared by adding $100 \mathrm{ml}$ of sterile de-ionized dist. water to 20 grams of the powder in sterile widemouth screw-capped bottles (200 $\mathrm{ml}$ volume), allowed to soak for 24 hours at $5^{\circ} \mathrm{C}$, then the mixture was centrifuged at $3000 \mathrm{rpm}$ for 10 minute and the supernatants were filtered. Curcumin aqueous extract was prepared by boiling $10 \%$ curcumin powder solution using sterile dist. water, left for sedimentation and then filtered. (Murad, 1998). Cumin and N. sativa cold pressed oils were purchased from local market.

\section{Preparation of Damietta cheese (Abou-Donia, 1991):}

Cheeses were manufactured in Dairy Technology Unit, Fac. Agriculture, Suez Canal Univ., Ismailia., Egypt. Two quantities (30 litters each) of good quality raw cow's milk obtained from Fac. Vet. Med. Farm, Suez Canal Univ., Ismailia, Egypt, were thoroughly strained using clean, dry and sterile gauze into sterile double-jacketed vats. The milk was heated to $45^{\circ} \mathrm{C}$ and salted using clean, fine and dry salt to give final concentrations of $5 \%$ and $10 \%$, where the salt was dissolved in a small part of milk and then mixed with original milk. Rennet extract (Powder) was added in appropriate concentration. Each quantity of milk was thoroughly divided into six parts (each 5 liter), the first part was taken as control (untreated) and the other five parts were treated with chosen concentrate of different spices according to the previous recommended studies which reveal the most antibacterial activity with acceptable flavors. The first part was treated with $3 \%$ bitter cumin aqueous extract (Megahed and Hafez, 2012), the second part with $3 \% \mathrm{~N}$. sativa water extract (Alsawaf and Alnaemi, 2011), the third part with $0.3 \%$ curcumin aqueous extract (Hosny et al., 2011), the fourth part with $0.3 \%$ bitter cumin oil (Omar, 2014) and the fifth part with $0.3 \% \mathrm{~N}$. sativa cold pressed oil (Alsawaf and Alnaemi, 2012). The treated milks and control one were kept at $45^{\circ} \mathrm{C}$ for 3 hours until curd formation and then the curd was pressed in a stainless steel mold lined with a clean sterile cloth for 12 hours. The finished curd was preserved in its whey in refrigerator at $5^{\circ} \mathrm{C} \pm 1$. Control and treated cheese samples were subjected for sensory and microbiological evaluation at times $0,7,14,21$ and 30 days.

\section{Sensory evaluation}

Damietta cheese samples were subjected for sensory evaluation (taste \& odor and color \& appearance). The evaluation was carried out by 10 untrained panelists compromising staff members and postgraduate students in Food Hygiene Department, Fac. Vet. Med., Suez Canal Univ, Egypt, to judge the sensory attributes according to Lawless and Hildegrade (2010). They were informed and trained to understand the used words and sensory scores.

\section{Microbiological evaluation}

Tenth-fold serial dilutions were prepared using sterile peptone water (APHA, 2004). Microbial groups were enumerated including total bacterial count (TBC) using standard plate count agar (Merck, Art. Nr., 1621), Enterobactriacae count using double layer of violet red bile glucose agar (Merck, Art. Nr., 1406), Enterococci count using Kanamycin Escculin Azide agar (Merck, Art. Nr., 5222) and Yeast and mold count (YMC) using yeast extract chloramphenicol glucose agar (Merck, Art. Nr., 16000) according to APHA (2004). S.aureus was enumerated using Baired-Parker agar (CM0275- Oxoid, Hampshire, England) according to Finegold and Martin (1982). Fungal growth was visually observed at 2 days interval on cheese surfaces and whey of both control and treated Damietta cheese.

\section{Statistical analysis}

Data were analyzed using the two-ways analysis of variance (ANOVA) according to the general linear model procedures. Logarithmic transformations were applied for all microbiological counts. Mean separations were done through Duncan's Multiple Range Test using the Statistical Analysis System, SAS 9.2, (SAS Institute, Inc., 2009). Results were considered statistically significant at $(\mathrm{P} \leq 0.05)$.

\section{RESULTS}

The results of sensory and microbiological quality of control and treated Damietta cheeses were represented in Tables $1-6$. 


\section{Assiut Vet. Med. J. Vol. 61 No. 147 October 2015}

Table 1: The sensory evaluation of control and treated cheeses.

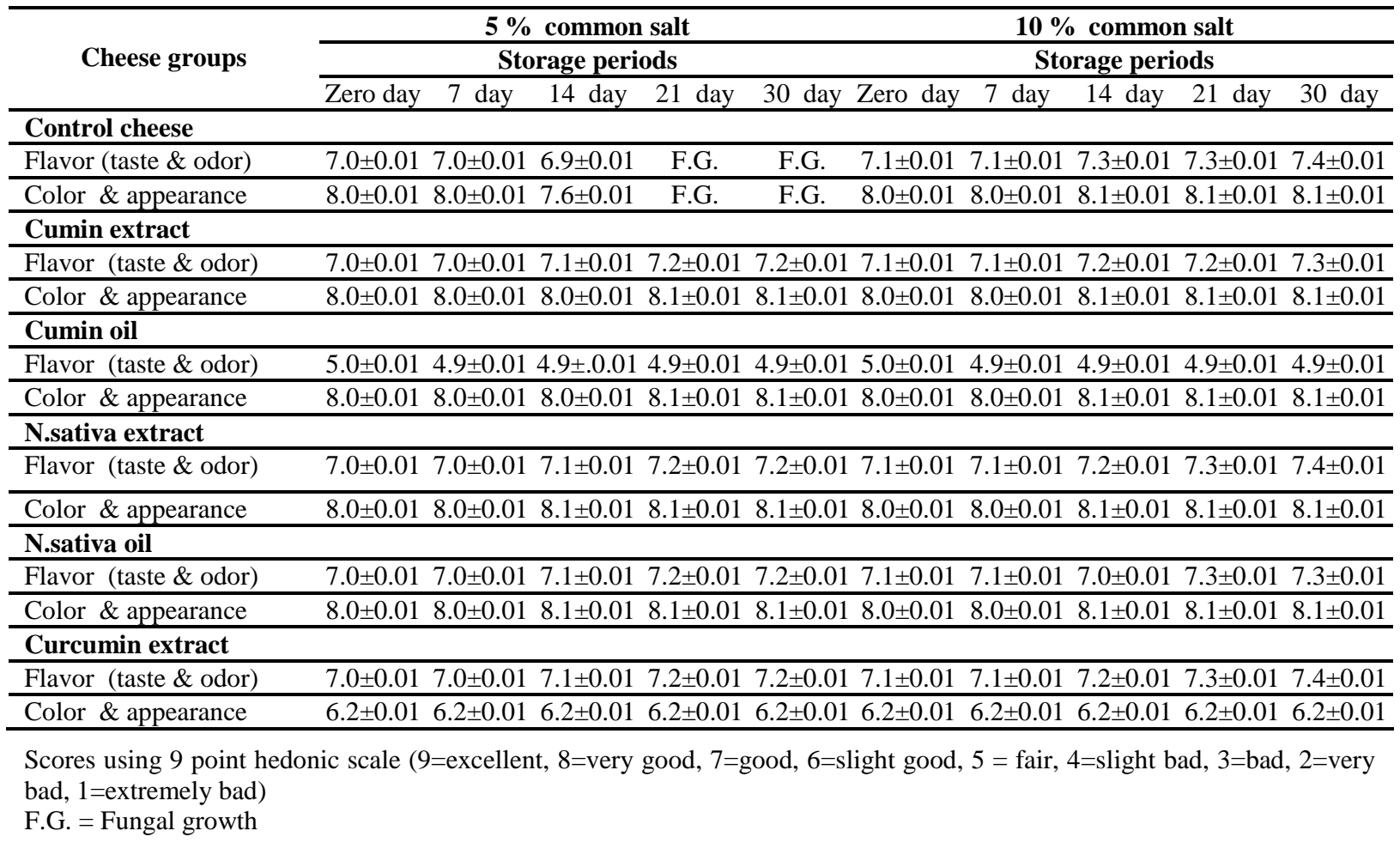

Table 2: The effect of spices on Logarithmic total bacterial count in Damietta cheese with special reference to its effect 1) among groups; 2) storage periods; 3) salt conc.; 4) salt conc. and storage periods and 5) salt conc. and groups.

\begin{tabular}{|c|c|c|c|c|c|c|c|c|c|c|c|}
\hline \multirow[t]{2}{*}{ 1. Cheese groups } & \multicolumn{5}{|c|}{$5 \%$ common salt } & \multicolumn{5}{|c|}{$10 \%$ common salt } & \multirow[b]{2}{*}{ Total group } \\
\hline & Zero d & $7 d$ & $14 \mathrm{~d}$ & $21 \mathrm{~d}$ & $30 \mathrm{~d}$ & Zero d & $7 d$ & $14 \mathrm{~d}$ & $21 \mathrm{~d}$ & $30 \mathrm{~d}$ & \\
\hline Control & 6.90308 & 6.97313 & 7.86332 & 7.94448 & 8.96379 & 6.79885 & 6.82276 & 6.89139 & 7.10848 & 7.32607 & $7.39253706^{\mathrm{a}}$ \\
\hline Cumin extract & 6.89763 & 6.73308 & 5.59106 & 4.32221 & 4.24139 & 6.83885 & 5.66275 & 4.60443 & 4.10848 & 4.00607 & $6.30329531^{\mathrm{b}}$ \\
\hline Cumin oil & 6.78533 & 6.51851 & 5.99563 & 5.74036 & 4.50515 & 6.50514 & 5.38021 & 4.07918 & 3.83251 & 3.95904 & $5.33010844^{\circ}$ \\
\hline N.sativa extract & 6.91907 & 6.57506 & 6.30309 & 5.83885 & 5.83251 & 6.07918 & 6.07432 & 5.11763 & 4.61278 & 4.59106 & $5.93345744^{\mathrm{bc}}$ \\
\hline N.sativa oil & 6.83885 & 6.26848 & 6.14509 & 5.86332 & 4.00000 & 6.36172 & 6.33251 & 5.13978 & 3.79934 & 3.36172 & $5.44508417^{\mathrm{b}}$ \\
\hline \multirow[t]{2}{*}{ Curcumin extract } & 6.89209 & 6.83885 & 5.75587 & 4.90848 & 4.79934 & 6.32223 & 5.85126 & 4.47712 & 4.32221 & 3.68124 & $5.38487035^{\circ}$ \\
\hline & & & & & & & & & & & $\begin{array}{c}\text { p-value }= \\
0.001\end{array}$ \\
\hline \multirow{2}{*}{$\begin{array}{l}\text { 2. Storage } \\
\text { periods }\end{array}$} & \multicolumn{2}{|c|}{ Zero day } & \multicolumn{2}{|c|}{7 day } & \multicolumn{2}{|c|}{14 day } & \multicolumn{2}{|c|}{21 day } & \multicolumn{2}{|c|}{30 day } & \multirow{2}{*}{$\begin{array}{c}\text { p-value }= \\
0.001\end{array}$} \\
\hline & 6.681 & $83710^{\mathrm{a}}$ & 6.4376 & $56242^{\mathrm{a}}$ & 6.0058 & $8201^{\mathrm{ab}}$ & 5.583 & $46202^{\mathrm{bc}}$ & 5.1156 & $51705^{\mathrm{c}}$ & \\
\hline \multirow[t]{2}{*}{ 3. Salt conc. } & \multicolumn{4}{|c|}{$5 \%$ salt } & & \multicolumn{5}{|c|}{$10 \%$ salt } & \multirow{2}{*}{$\begin{array}{c}\text { p-value }= \\
0.013\end{array}$} \\
\hline & \multicolumn{4}{|c|}{$6.26757270^{\mathrm{a}}$} & & \multicolumn{5}{|c|}{$5.66221155^{\mathrm{b}}$} & \\
\hline \multirow{2}{*}{$\begin{array}{l}\text { 4. Salt conc. } \\
\text { *storage periods }\end{array}$} & \multicolumn{4}{|c|}{$5 \%$ zero $5 \% * 7 \mathrm{~d} 5 \% * 14 \mathrm{~d} 5 \% * 21 \mathrm{~d}$} & $5 \% 30 \mathrm{~d}$ & $10 *$ zero & \multicolumn{4}{|c|}{$10 \% * 7 \mathrm{~d} 10 \% * 14 \mathrm{~d} 10 \% * 21 \mathrm{~d} 10 \% * 30 \mathrm{~d}$} & \multirow{2}{*}{$\underset{0.011}{p-v a l u e}=$} \\
\hline & $6.87268^{\mathrm{a}}$ & $6.846187^{\mathrm{a}}$ & $6.49234^{\mathrm{b}}$ & $5.76962^{\mathrm{c}}$ & $5.35702^{\mathrm{d}}$ & $6.49099^{\mathrm{b}}$ & $6.02913^{\mathrm{b}}$ & $5.51942^{\mathrm{c}}$ & $5.39730^{\text {cd }}$ & $4.87420^{\mathrm{e}}$ & \\
\hline \multicolumn{12}{|c|}{ 5. Cheese groups * salt concentration } \\
\hline & \multicolumn{2}{|c|}{ Control } & \multicolumn{2}{|c|}{ Cumin extract } & \multicolumn{2}{|c|}{ Cumin oil } & \multicolumn{2}{|c|}{ N.sativa extract } & \multicolumn{2}{|c|}{ N.sativa oil } & $\begin{array}{c}\text { Curcumin } \\
\text { extract }\end{array}$ \\
\hline $5 \%$ salt & \multicolumn{2}{|c|}{$7.72956^{\mathrm{a}}$} & \multicolumn{2}{|c|}{$5.55107^{\mathrm{cd}}$} & \multicolumn{2}{|c|}{$5.90899^{\mathrm{bcd}}$} & \multicolumn{2}{|c|}{$6.47372^{\mathrm{abc}}$} & \multicolumn{2}{|c|}{$6.10315^{\mathrm{bcd}}$} & $5.83892^{\text {bcd }}$ \\
\hline $10 \%$ salt & 7.05 & $551^{\mathrm{ab}}$ & 7.055 & $551^{\mathrm{ab}}$ & 4.75 & $122^{\mathrm{d}}$ & 5.39 & $319^{\mathrm{cd}}$ & 4.78 & $701^{\mathrm{d}}$ & $4.93081^{\mathrm{d}}$ \\
\hline & & & & & p-value & $=0.023$ & & & & & \\
\hline
\end{tabular}

Log counts with different superscripts showed highly significant differences at $(\mathrm{P} \leq 0.01)$

The original counts can be obtained by the antilogarithm (Base 10) of these results. 
Table 3: The effect of spices on Logarithmic total Enterobacteriaceae count in Damietta cheese with special reference to its effect 1) among groups; 2) storage periods; 3) salt conc.; 4) salt conc. and storage periods, and 5) salt conc. and groups.

\begin{tabular}{|c|c|c|c|c|c|c|c|c|c|c|c|}
\hline \multirow[t]{2}{*}{ 1. Cheese groups } & \multicolumn{5}{|c|}{$5 \%$ common salt } & \multicolumn{5}{|c|}{$10 \%$ common salt } & \multirow[b]{2}{*}{ Total group } \\
\hline & Zero d & $7 \mathrm{~d}$ & $14 \mathrm{~d}$ & $21 \mathrm{~d}$ & $30 \mathrm{~d}$ & Zero d & $7 d$ & $14 \mathrm{~d}$ & $21 \mathrm{~d}$ & $30 \mathrm{~d}$ & \\
\hline Control & 4.49136 & 4.59106 & 5.47712 & 5.53147 & 5.77815 & 4.43136 & 4.51954 & 4.56239 & 4.76332 & 4.7834 & $4.72092^{\mathrm{a}}$ \\
\hline Cumin extract & 4.59106 & 4.47712 & 4.43136 & 4.37712 & 3.36172 & 4.66275 & 4.74818 & 4.38021 & 3.72607 & 3.46239 & $4.34180^{\mathrm{abc}}$ \\
\hline Cumin oil & 4.61278 & 4.57978 & 4.31278 & 3.74818 & 3.77815 & 4.57978 & 4.54406 & 3.90848 & 2.78532 & 2.66646 & $3.97828^{\text {cd }}$ \\
\hline N.sativa extract & 4.59106 & 4.46239 & 4.39239 & 4.34509 & 3.82406 & 4.62324 & 4.25527 & 4.32221 & 3.98677 & 3.83250 & $4.42550^{\mathrm{ab}}$ \\
\hline N.sativa oil & 4.95424 & 4.95424 & 4.90309 & 3.74036 & 3.57978 & 4.60000 & 3.81954 & 3.78532 & 2.55630 & 2.25527 & $3.84975^{\mathrm{d}}$ \\
\hline \multirow[t]{2}{*}{ Curcumin extract } & 4.54406 & 4.47712 & 4.16848 & 3.82941 & 3.65904 & 4.37875 & 4.24242 & 3.86275 & 3.65733 & 2.69019 & $4.17095^{\text {bcd }}$ \\
\hline & & & & & & & & & & & p-value $=0.002$ \\
\hline \multirow{2}{*}{$\begin{array}{l}\text { 2. Storage } \\
\text { periods }\end{array}$} & \multicolumn{2}{|c|}{ Zero day } & \multicolumn{2}{|c|}{7 day } & \multicolumn{2}{|c|}{14 day } & \multicolumn{2}{|c|}{21 day } & \multicolumn{2}{|c|}{30 day } & p-value $=0.001$ \\
\hline & \multicolumn{2}{|c|}{$4.52582^{\mathrm{a}}$} & \multicolumn{2}{|c|}{$4.50589^{\mathrm{a}}$} & \multicolumn{2}{|c|}{$4.47555^{\mathrm{a}}$} & \multicolumn{2}{|c|}{$4.01223^{\mathrm{b}}$} & \multicolumn{2}{|c|}{$3.71984^{\mathrm{b}}$} & \\
\hline \multirow[t]{2}{*}{ 3. Salt conc. } & \multicolumn{5}{|c|}{$5 \%$ salt } & \multicolumn{5}{|c|}{$10 \%$ salt } & p-value $=0.001$ \\
\hline & \multicolumn{4}{|c|}{$4.59280^{\mathrm{a}}$} & \multicolumn{6}{|c|}{$3.90293^{\mathrm{b}}$} & \\
\hline \multirow{2}{*}{$\begin{array}{l}\text { 4. Salt conc. } \\
\text { *storage periods }\end{array}$} & $5 \%$ zero & $5 \% * 7 d$ & $5 \% * 14 \mathrm{~d}$ & $5 \% * 21 d$ & $5 \% 30 \mathrm{~d}$ & $10 *$ zero & $10 \% * 7 \mathrm{~d}$ & $10 \% * 14 d$ & $10 \% * 21 \mathrm{~d}$ & $10 \% * 30 \mathrm{~d}$ & $p$-value $=0.034$ \\
\hline & $4.63076^{\mathrm{ab}}$ & $4.59028^{\mathrm{ab}}$ & $4.86420^{\mathrm{a}}$ & $4.54527^{\mathrm{ab}}$ & $4.33348^{\mathrm{ab}}$ & $4.42088^{\mathrm{ab}}$ & $4.42150^{\mathrm{ab}}$ & $4.08690^{\mathrm{b}}$ & $3.47918^{\mathrm{c}}$ & $3.10621^{\mathrm{c}}$ & \\
\hline \multicolumn{12}{|c|}{ 5. Cheese groups * salt concentration } \\
\hline & \multicolumn{2}{|c|}{ Control } & \multicolumn{2}{|c|}{ Cumin extract } & \multicolumn{2}{|c|}{ Cumin oil } & \multicolumn{2}{|c|}{ N.sativa extract } & \multicolumn{2}{|c|}{ N.sativa oil } & Curcum extract \\
\hline $5 \%$ salt & \multicolumn{2}{|c|}{$5.17383^{\mathrm{a}}$} & \multicolumn{2}{|c|}{$4.46767^{\mathrm{abcd}}$} & \multicolumn{2}{|c|}{$4.26633^{\mathrm{bcd}}$} & \multicolumn{2}{|c|}{$4.64700^{\mathrm{ab}}$} & \multicolumn{2}{|c|}{$4.42634^{\text {abcd }}$} & $4.57562^{\mathrm{abc}}$ \\
\hline $10 \%$ salt & 4.268 & $301^{\text {bed }}$ & 3.21 & $592^{\mathrm{b}}$ & 3.290 & $022^{\mathrm{de}}$ & 3.20 & $400^{\text {cd }}$ & 3.27 & $316^{\mathrm{e}}$ & $3.76629^{\text {cde }}$ \\
\hline & & & & & p-value $=$ & $=0.046$ & & & & & \\
\hline
\end{tabular}

Log counts with different superscripts showed significant differences at $(\mathrm{P} \leq 0.05)$.

The original counts can be obtained by the antilogarithm (Base 10) of these results.

Table 4: The effect of spices on Logarithmic total Enterococci count in Damietta cheese with special reference to its effect 1) among groups; 2) storage periods; 3) salt conc.; 4) salt conc. and storage periods and 5) salt conc. and groups.

\begin{tabular}{|c|c|c|c|c|c|c|c|c|c|c|c|}
\hline \multirow[t]{2}{*}{ 1. Cheese groups } & \multicolumn{5}{|c|}{$5 \%$ common salt } & \multicolumn{5}{|c|}{$10 \%$ common salt } & \multirow[b]{2}{*}{ Total group } \\
\hline & Zero d & $7 \mathrm{~d}$ & $14 \mathrm{~d}$ & $21 \mathrm{~d}$ & $30 \mathrm{~d}$ & Zero d & $7 \mathrm{~d}$ & $14 \mathrm{~d}$ & $21 \mathrm{~d}$ & $30 \mathrm{~d}$ & \\
\hline Control & 4.77815 & 4.81954 & 4.90848 & 5.41497 & 5.81497 & 4.79209 & 4.80848 & 4.87506 & 4.9218 & 4.97136 & $4.35823^{\mathrm{a}}$ \\
\hline Cumin extract & 4.89209 & 4.83885 & 4.77815 & 4.68124 & 3.98677 & 4.83884 & 4.85630 & 4.58649 & 3.95904 & 2.92941 & $4.43472^{\mathrm{a}}$ \\
\hline Cumin oil & 4.93952 & 4.25424 & 3.92427 & 3.25424 & 2.98677 & 4.86172 & 4.38081 & 3.64345 & 2.36172 & 2.25527 & $3.80620^{\mathrm{bc}}$ \\
\hline N.sativa extract & 4.93449 & 4.84509 & 4.69897 & 4.15125 & 3.34242 & 4.83239 & 4.71291 & 3.62324 & 2.44509 & 2.04139 & $3.97272^{\mathrm{ab}}$ \\
\hline N.sativa oil & 4.65321 & 4.22427 & 3.67712 & 2.47209 & 2.01897 & 4.27875 & 3.59106 & 2.55242 & 2.34630 & 2.28346 & $3.36276^{\mathrm{c}}$ \\
\hline \multirow[t]{2}{*}{ Curcumin extract } & 4.95424 & 4.78532 & 4.64345 & 3.86332 & 3.77815 & 4.74242 & 4.27875 & 3.62324 & 2.78532 & 2.56820 & $3.96224^{\mathrm{ab}}$ \\
\hline & & & & & & & & & & & p-value $=0.001$ \\
\hline \multirow{2}{*}{$\begin{array}{l}\text { 2. Storage } \\
\text { periods }\end{array}$} & \multicolumn{2}{|c|}{ Zero day } & \multicolumn{2}{|c|}{7 day } & \multicolumn{2}{|c|}{14 day } & \multicolumn{2}{|c|}{21 day } & \multicolumn{2}{|c|}{30 day } & p-value $=0.002$ \\
\hline & \multicolumn{2}{|c|}{$4.71649^{\mathrm{a}}$} & \multicolumn{2}{|c|}{$4.66630^{\mathrm{a}}$} & \multicolumn{2}{|c|}{$3.93536^{\mathrm{b}}$} & \multicolumn{2}{|c|}{$3.50198^{\mathrm{bc}}$} & \multicolumn{2}{|c|}{$3.09393^{\mathrm{c}}$} & \\
\hline \multirow[t]{2}{*}{ 3. Salt conc. } & \multicolumn{5}{|c|}{$5 \%$ salt } & \multicolumn{5}{|c|}{$10 \%$ salt } & p-value $=0.000$ \\
\hline & \multicolumn{4}{|c|}{$4.33316^{\mathrm{a}}$} & \multicolumn{6}{|c|}{$3.63247^{\mathrm{b}}$} & \\
\hline \multirow{2}{*}{$\begin{array}{l}\text { 4. Salt conc. } \\
\text { *storage periods }\end{array}$} & \multicolumn{3}{|c|}{$5 \%$ zero $5 \% * 7 \mathrm{~d} 5 \% * 14 \mathrm{~d}$} & $5 \% * 21 d$ & \multicolumn{2}{|c|}{$5 \% 30 \mathrm{~d} 10 *$ zero } & $10 \% * 7 \mathrm{~d} 1$ & $0 \% * 14 d$ & $10 \% * 21 d$ & $10 \% * 30 \mathrm{~d}$ & \multirow[t]{2}{*}{ p-value $=0.009$} \\
\hline & $4.85862^{\mathrm{a}}$ & $4.82788^{\mathrm{a}}$ & $4.20507^{\mathrm{b}}$ & $4.23952^{\mathrm{b}}$ & $3.53467^{\mathrm{c}}$ & $4.57437^{\mathrm{ab}}$ & $4.50472^{\mathrm{ab}}$ & $3.66565^{\mathrm{c}}$ & $2.76444^{\mathrm{d}}$ & $2.65318^{d}$ & \\
\hline \multicolumn{12}{|c|}{ 5. Cheese groups * salt concentration } \\
\hline & \multicolumn{3}{|c|}{ Control } & Cumin extract & \multicolumn{2}{|c|}{ Cumin oil } & \multicolumn{3}{|c|}{ N.sativa extract } & N.sativa oil & Curcumin extract \\
\hline $5 \%$ salt & \multicolumn{3}{|c|}{$4.66722^{\mathrm{a}}$} & $4.635421^{\mathrm{a}}$ & \multicolumn{2}{|c|}{$4.11181^{\mathrm{b}}$} & \multicolumn{3}{|c|}{$4.53444^{\mathrm{ab}}$} & $3.64513^{\mathrm{bc}}$ & $4.40489^{\mathrm{ab}}$ \\
\hline \multirow[t]{2}{*}{$10 \%$ salt } & & $4.04923^{\mathrm{b}}$ & & $4.23402^{\mathrm{b}}$ & 3.500 & $059^{\mathrm{bc}}$ & & $3.41100^{\mathrm{b}}$ & & $3.08040^{\mathrm{c}}$ & $3.51959^{\mathrm{bc}}$ \\
\hline & & & & & p-valt & lue $=0.0$ & & & & & \\
\hline
\end{tabular}

Log counts with different superscripts showed significant differences at $(\mathrm{P} \leq 0.05)$.

The original counts can be obtained by the antilogarithm (Base 10) of these results. 


\section{Assiut Vet. Med. J. Vol. 61 No. 147 October 2015}

Table 5: The effect of spices on Logarithmic total yeast and mould count in Damietta cheese with special reference to its effect 1) among groups; 2) storage periods; 3) salt conc.; 4) salt conc. and storage periods and 5) salt conc. and groups.

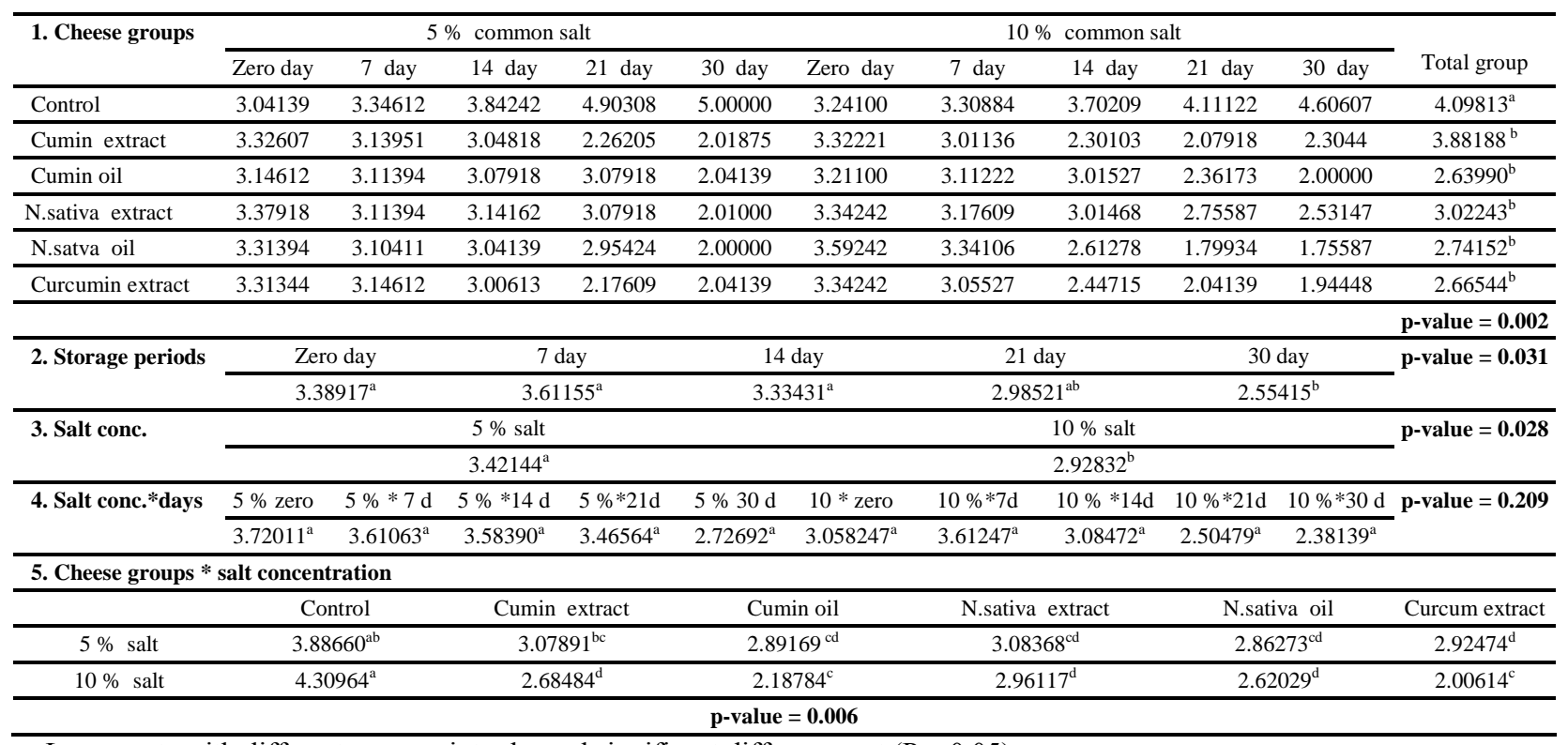

Log counts with different superscripts showed significant differences at $(\mathrm{P} \leq 0.05)$.

The original counts can be obtained by the antilogarithm (Base 10) of these results.

Table 6: The effect of spices on Logarithmic S.aureus count in Damietta cheese with special reference to its effect 1) among groups; 2) storage periods; 3) salt conc.; 4) salt conc. and storage periods and 5) salt conc. and groups.

Log counts with different superscripts showed significant differences at $(\mathrm{P} \leq 0.05)$.

\begin{tabular}{|c|c|c|c|c|c|c|c|c|c|c|c|}
\hline \multirow[t]{2}{*}{ 1. Cheese groups } & \multicolumn{5}{|c|}{$5 \%$ common salt } & \multicolumn{5}{|c|}{$10 \%$ common salt } & \multirow[b]{2}{*}{ Total group } \\
\hline & Zero d & $7 \mathrm{~d}$ & $14 \mathrm{~d}$ & $21 \mathrm{~d}$ & $30 \mathrm{~d}$ & Zero d & $7 \mathrm{~d}$ & $14 \mathrm{~d}$ & $21 \mathrm{~d}$ & $30 \mathrm{~d}$ & \\
\hline Control & 3.41497 & 3.46239 & 3.51851 & 4.47712 & 4.71600 & 3.38021 & 3.66607 & 3.82275 & 3.90845 & 3.99934 & $3.91658^{\mathrm{a}}$ \\
\hline Cumin extract & 3.65978 & 3.57321 & 3.49136 & 2.45527 & 2.30103 & 3.61278 & 3.32221 & 2.99563 & 2.77609 & 2.25527 & $2.96426^{\mathrm{b}}$ \\
\hline Cumin oil & 3.49136 & 3.44715 & 2.61278 & 1.95424 & 0 & 3.46239 & 2.04139 & 1.06332 & 0 & 0 & $2.55323^{\mathrm{bc}}$ \\
\hline N.sativa extract & 3.37103 & 3.32221 & 3.25527 & 3.00000 & 2.54139 & 3.80172 & 3.10617 & 2.32221 & 2.07918 & 1.89209 & $2.73813^{\mathrm{bc}}$ \\
\hline N.sativa oil & 3.50514 & 2.44715 & 2.38021 & 1.47712 & 0 & 3.24139 & 2.46239 & 1.99122 & 0 & 0 & $2.47209^{\mathrm{bc}}$ \\
\hline \multirow[t]{2}{*}{ Curcumin extract } & 3.32222 & 2.57978 & 2.07918 & 1.00000 & 0 & 3.10000 & 2.15527 & 2.00000 & 0 & 0 & $2.31949^{c}$ \\
\hline & & & & & & & & & & & p-value $=0.001$ \\
\hline \multirow[t]{2}{*}{ 2. Storage periods } & \multicolumn{2}{|c|}{ Zero day } & \multicolumn{2}{|c|}{7 day } & \multicolumn{2}{|c|}{14 day } & \multicolumn{2}{|c|}{21 day } & \multicolumn{2}{|c|}{30 day } & $p$-value $=0.003$ \\
\hline & \multicolumn{2}{|c|}{$3.37275^{\mathrm{a}}$} & \multicolumn{2}{|c|}{$3.05212^{\mathrm{ab}}$} & \multicolumn{2}{|c|}{$2.68104^{\mathrm{bc}}$} & \multicolumn{2}{|c|}{$2.36972^{\mathrm{c}}$} & \multicolumn{2}{|c|}{$2.83418^{\mathrm{bc}}$} & \\
\hline \multirow[t]{2}{*}{ 3. Salt conc. } & \multicolumn{5}{|c|}{$5 \%$ salt } & \multicolumn{5}{|c|}{$10 \%$ salt } & $\mathrm{p}$-value $=0.057$ \\
\hline & \multicolumn{4}{|c|}{$2.96614^{\mathrm{a}}$} & \multicolumn{6}{|c|}{$2.81323^{\mathrm{a}}$} & \\
\hline \multirow{2}{*}{$\begin{array}{l}\text { 4. Salt conc. } \\
\text { *storage periods }\end{array}$} & \multicolumn{2}{|c|}{$5 \%$ zero $5 \% * 7 \mathrm{~d}$} & $5 \% * 14 \mathrm{~d}$ & $5 \% * 21 \mathrm{~d}$ & $5 \% 30 \mathrm{~d}$ & $10 *$ zero & $10 \% * 7 \mathrm{~d}$ & $10 \% * 14 d$ & $10 \% * 21 d$ & $10 \% * 30 \mathrm{~d}$ & \multirow[t]{2}{*}{$\mathrm{p}$-value $=0.201$} \\
\hline & $3.43575^{\mathrm{a}}$ & $3.15198^{\mathrm{a}}$ & $2.88954^{\mathrm{a}}$ & $2.36062^{\mathrm{a}}$ & $3.0194^{\mathrm{a}}$ & $3.30975^{\mathrm{a}}$ & $2.95225^{\mathrm{a}}$ & $2.47252^{\mathrm{a}}$ & $2.38791^{\mathrm{a}}$ & $2.64890^{\mathrm{a}}$ & \\
\hline \multicolumn{12}{|c|}{ 5. Cheese groups * salt concentration } \\
\hline & \multicolumn{2}{|c|}{ Control } & \multicolumn{2}{|c|}{ Cumin extract } & \multicolumn{2}{|c|}{ Cumin oil } & \multicolumn{2}{|c|}{ N.sativa extract } & \multicolumn{2}{|c|}{ N.sativa oil } & Curcumin extract \\
\hline $5 \%$ salt & \multicolumn{2}{|c|}{$3.91780^{\mathrm{a}}$} & \multicolumn{2}{|c|}{$3.05613^{\mathrm{a}}$} & \multicolumn{2}{|c|}{$2.87638^{\mathrm{a}}$} & \multicolumn{2}{|c|}{$2.98398^{\mathrm{a}}$} & 2.45 & $241^{\mathrm{a}}$ & $2.24529^{\mathrm{a}}$ \\
\hline $10 \%$ salt & 3.91 & $537^{\mathrm{a}}$ & 2.872 & $40^{\mathrm{a}}$ & 2.12 & $237^{\mathrm{a}}$ & 2.4 & $228^{\mathrm{a}}$ & 2.49 & $833^{\mathrm{a}}$ & $2.41842^{\mathrm{a}}$ \\
\hline
\end{tabular}

The original counts can be obtained by the antilogarithm (Base 10) of these results. 


\section{DISCUSSION}

Weak organic acids are the most classical preservative agents, but now there has been increase concern of consumers about food free or with lower level of chemical preservatives, because these could be toxic for human. So, natural antimicrobial preservatives are used in food industry either to control natural spoilage of food, or to control disease producing organisms. Other benefits are decreasing the development of antibiotic resistance pathogens or strengthening immune system of human being (Gaysinsky and Weiss, 2007).

Cheeses treated with aqueous extract of cumin, N.sativa and curcumin were gave unchanged flavors (taste and odor) during the first two weeks. As the ripening period progress, the flavor characteristics were gradually increased leading to improvement of organoleptic properties, compared with control one. These findings were in agreement with Hosny et al. (2011); Samir et al. (2013) and Hassanein et al. (2014). On contrary, cheese treated with cumin oil gave slightly pronounced flavor of fair quality (score: 5.0 vs. 7.0). Similar findings were reported by $\mathrm{Li}$ and Jiang (2004), who proved that cumin oil has a distinctive flavor and strong warm aroma due to the essential oil contents. Moreover, the curcumin treated cheese showed faint-yellow color of slight good appearance (score: 6.2 vs 8.0 ); these findings were run parallel to those reported by Hosny et al. (2011).

Concerning the microbiological quality of cheeses, cumin oil showed a stronger antimicrobial effect than cumin aqueous extract leading to rapid reduction of the microbial groups in treated cheeses, compared with initial count ( 0 day), These findings were run parallel to that obtained by Dorman and Deans (2000), who reported that essential oils have strong effect than spices itself or its aqueous extract. The antibacterial activity of bitter cumin could be attributed to a number of polyphenolic compounds including gallic acid, protocatechuic acid, caffeic acid, ellagic acid, ferulic acid, as well as flavonoid compounds such as quercetin and kaempferol (Ani et $a l ., 2006)$. Also, they proved in an experiment that Gram-positive bacteria were found to be more sensitive to bitter cumin extracts than Gram-negative bacteria. The bacterial species $B$. subtilis, B. cereus and $S$. aureus were found to be highly sensitive and showed a significant inhibition of the growth in the presence of bitter cumin extract. Enterobacter spp. and L. monocytogenes were moderately inhibited, while E. coli and $Y$. enterocolitica were resistant to bitter cumin extract. They explained that the antibacterial activity of flavonoids was attributed to the inhibition of the synthesis of DNA and RNA and other related macromolecules. Further, it was found that phenolic compounds with more than three $3-\mathrm{OH}$ possess antibacterial activity.
N.sativa oil was more effective than N. sativa aqueous extract causing rapid reduction to all microbial groups in treated cheeses, compared with initial count ( 0 day). The obtained results go hand to hand with those reported by Wahba et al. (2004); Badawi et al. (2009) and Alsawaf and Alnaemi (2011), who recorded a rapid reduction of microbial counts in soft cheese containing N.sativa seed and oil added during preparation. Burits and Bucar (2000) attributed the microbial reduction to presence of Thymoquinone TQ (2-isopropyl-5-methylbenzoquinone) which was considered as one of the major components of N.sativa volatile oils and fixed oils. Kahasi (2002) explained that antimicrobial effect of TQ was related to the inhibition of RNA and protein synthesis. In addition, the unsaturated bicyclic monoterpene hydrocarbon which also present in $\mathrm{N}$. sativa oil exerts antibacterial action. Therefore, bacterial types present in cheeses treated with $\mathrm{N}$. sativa oil will be more exposed to the antibacterial action than those present in cheeses treated with $\mathrm{N}$. sativa seed. Ahn et al. (2004) found that the antimicrobial action of bioactive compounds in N.sativa oil which mainly contain phenolic compounds cause bacteriostatic and antiproliferative actions against bacterial growth. Luther et al. (2007) proved that N.sativa oil characterized by high level of phenolics which are considered as powerful active compounds expressing strong antimicrobial activities. Bettaieb et al. (2010) attributed this activity mainly to their redox potential, which can play an important role in adsorbing and neutralizing free radicals and chelating metals, especially iron and copper cations.

Curcumin extract was found to be the most effective additives among the aqueous spices extract used giving a rapid reduction to all microbial groups in treated cheeses, compared with initial counts. The obtained results were in agreement with those reported by Murad (1998); Tajbakash et al. (2008) and Hosny et al. (2011), who found that Curcumin has wide spectra of antibacterial activity against Gram-negative and Gram-positive pathogenic or nonpathogenic bacteria in soft cheese. The antibacterial activity of Curcumin was related to the presence of compounds belonging to flavonoids and terpens particularly to borneol, cymene, cuparene, ptolymethyl-carbinol, curcumin and careen (Thongson et al., 2005). In addition, Curcumin has wide spectra of biological actions such as anti-inflammatory (Puntihavathi et al., 2000), antifungal activities, (Chatopadhyay et al., 2004), antidiabetic (Lotempio et al., 2005), antiviral (Suzuki et al., 2005), antiallergic (Aggarwal et al., 2007) and anticancer (Menon and sudheer, 2007). Curcumin contains a mixture of powerful antioxidant phytonutrients known as Curcuminoids that inhibits cancer at initiation, promotion and progression stages of tumor development. Also, it is a strong anti-oxidant which supports colon health, exerts neuroprotective activity 
and helps to maintain a healthy cardiovascular system (Siddiqui et al., 2006).

Regarding the comparison between both types of control and treated cheeses, the microbial groups showed rapid multiplication rates in control cheeses of $5 \%$ salt, compared with control one of $10 \%$ salt along the storage periods. These findings agree with those obtained by Alsawaf and Alnaemi (2011), who found that control cheese of $10 \%$ salt revealed slightly increase in microbial counts which may be attributed to the high concentration of sodium chloride used in manufacturing. On contrary, the reduction rates of the microbial groups were rapid in treated cheeses of $10 \%$ salt, compared with $5 \%$ salt, these findings explained the role of spices in improvement the microbial quality of cheeses especially that manufactured from raw milk. Also, there was a high significant difference for the microbial groups between treated cheeses and control one (p-value $0.001-0.002$ ). These findings were run parallel to that reported by Hayaloglu and Farkye (2011), who attributed that to the combined effect of salt and spices extract.

With regard to the effect of storage periods on the microbial groups tested, the statistical results point out that TBC, Enterobactericeaea and Enterococci, compared with 0 day, showed highly significant reduction ( $\mathrm{p}$-values $0.001-0.003$ ), while YMC showed a significant reduction (p-value 0.031 ) in both types ( $5 \& 10 \%$ salt) of treated cheeses.

According to the effect of salt concentration on the microbial groups, the total results indicated that both TBC and YMC showed significant reduction ( $\mathrm{p}$-value $\leq$ 0.05), while both Enterobactericeae and Enterococci showed high significant reduction (pvalue 0.001), whereas S.aureus showed no significance (p-value 0.57). Concerning salt concentration and cheese groups, there was a difference between cheeses of $5 \%$ and $10 \%$ salt in all cheese groups ranged from significant ( $p$-value $\leq$ 0.05 ) to high significant ( $p$-value 0.006 ) for all the affected microbial groups, except S.aureus that showed un significant difference ( $p$-value 0.120). These findings were supported by Kang and Park (1984), who found that the high concentration of sodium chloride $(10 \%)$ probably inhibits the growth of many microorganisms by raising the osmotic pressure of the brine leading to migration of water inside the microbial cell through the membrane into the brine causing a partial dehydration of the cell and consequently slows metabolic processes resulting in interference with the multiplication of the microorganisms (Sumner et al., 1990). The failure of high salt content to affect S.aureus was probably attributed to that the organism was salt tolerant (Bruins et al., 2007).
The fungal growth were observed at 21 days of storage over cheese surface and whey in control cheese of $5 \%$ salt only, The obtained findings explained the role of spices in inhibiting the fungal growth in soft cheese of low salt content. These results were supported by those obtained by Murad (1998), who found that some natural extracts can control yeast growth in yoghurt. Also, the absence of fungal growth in control cheese of high salt content $(10 \%)$ and in other treated cheeses along the storage periods supported these findings.

It could be conclude that addition of the selected spices to soft cheese will improve its sensory and microbial quality, moreover, some of spices can eliminate S.aureus, as food poisoning microorganisms, from soft cheeses. Therefore, cheese contained natural compounds may become an important approach for many consumers, to achieve their desires, to reduce the risk of diseases and to treat minor illnesses. Also, the uses of essential oils have strong antimicrobial effect than the spices itself or its aqueous extract.

\section{REFERENCES}

Abou-Donia, S.A. (1991): Manufacture of Egyptian of soft and pickled cheese. Chapter 5: Feta and Retaled cheeses, Ed. By Robinsons RK and Tamine AY, Published by Woodhead Publishing Limited, Cambridge, England.

Aggarwal, B.B.; Sundarma, C.; Malani, N.M. and Ichikawa, H. (2007): Curcumin: the Indian solid gold. Adv. Exp. Med Biol. 595: 1-75.

Ahn, J.; Grun, I.U. and Mustaph, A. (2004): Antimicrobial and antioxidant activities of natural extracts in vitro and in ground beef. $\mathrm{J}$. Food Prot. 67: 148-155.

Alsawaf, S.D. and Alnaemi, H.S. (2011): Effect of Nigella sativa (seed and oil) on the bacteriological quality of soft white cheese. Iraqi J. Veterinary Science, 25: 21-27.

American Public Health association "APHA" (2004): Compendium of Methods for the Microbiological Examination of Food. 17th ed, APHA, Washington D. C. USA.

Ani, V.; Varadaraj, M.C. and Akhilender Naidu, K. (2006): Antioxidant and antibacterial activities of polyphenolic compounds from bitter cumin (Cuminum nigrum L.). Eur Food Res Technol. 224: 109-115.

Arora, D.S. and Kaur, J. (1999): Antimicrobial activity of spices. International Journal of Antimicrobial Agents. 12: 257-262.

Badawi, S.K.H.; Al-Badrany, H.S. and Hasan, G.M. (2009): Using of essential oils extracted from some spices to extend the shelf life of soft white cheese. Mesopotamia J Agri. 37: 125-130. 
Bettaieb, I.; Bourgou, S.; Wannes, W.A.; Hamrouni, I.; Limam, F. and Marzouk, B. (2010): Essential oils, phenolics, and antioxidant activities of different parts of cumin (Cuminum cyminum L.). J. Agric. Food Chem. 58: 10410-10418.

Bruins, M.J.; Juffer P.; Maurice, J.H.; Wolfhagen, M.; Gijs, J.H. and Ruijs, M. (2007): Salt Tolerance of Methicillin-Resistant and Methicillin-Susceptible and Staphylococcus aureus. Journal of Clinical Microbiology, 45: 682-683.

Burits, M. and Bucar, F. (2000): Antioxidant activity of Nigella sativa oil. Phytother Res. 14: 323-328.

Chatopadhyay, I.; Biswas, I.; Bandyopadhyay, U. and Banerjee, R.K. (2004): Turmeric and Curcumin: Biological actions and medicinal applications. Curr.Sci. 87: 44-53.

Dormans, H.J. and Deans, S.G. (2000): Antimicrobial agents from plants: antimicrobial activity of plant volatiles oil, J. Al. Microbiol., 88: 308-316.

Finegold, S.M. and Martin, W.J. (1982): Bailley and Scott Diagnostic Microbiology. 6th Edn., C.V. Mosby Co.St. Louis, Toronto, London.

Gaysinsky, S. and Weiss, J. (2007): Aromatic and spice plants: Uses in food safety. Stewart Post Harvest Review, 4: 1-9.

Hassanien, M.F.R.; Samir A.M. and Kahled, M. (2014): Soft cheese supplemented with black cumin oil: Impact on food borne pathogens and quality during storage. Saudi Journal of Biological Sciences, 21: 280-288.

Hayaloglu, A.A. and Farkye, N.Y. (2011): Cheese with added Herbs, Spices and Condiments. Encyclopedia of Dairy Science (Second Edition), 783-789.

Hosny I.M.; El Kholy, W.I.; Murad, H.A. and El Dairouty, R.K. (2011): Antimicrobial activity of Curcumin upon pathogenic microorganisms during manufacture and storage of a novel style cheese 'Karishcum". Journal of American Science, 7: 611-618.

Kahsai, A.W. (2002): Isolation and characterization of active ingredients from Nigella sativa for antibacterial screening. MSc (Chemistry), East Tennessee State University, USA.

Kang, J.H. and Park, Y.H. (1984): Effect of food additives on the histamine formation during processing and storage of mackerel. Bull. Korean Fish. Soc. 17: 383.

Kizil, S. and Sogut, T. (2003): investigation of antibacterial effects of spices. Crop Research, 3: 86-90.

Lawless, H.T. and Hildegrade, H. (2010): Sensory Evaluation of Food: Principles and Practice. $1^{\text {st }}$ Edn., Springer Publisher, ISBN-10: 1441964886, pp: 619.
Li, R. and Jiang, Z.T. (2004): Chemical composition of essential oil of Cuminum cyminum L. from China. Flavour and Fragrance Journal 19: 311-313.

Lotempio, M.M.; Veena, M.S.; Steel, H.L.; Ramamurthy, B. and Wang, M.B. (2005): Curcumin suppresses growth of head and neck squamous cell carcinoma. Clin. Cancer Res.11: 6994-7002.

Luther, M.; Parry, J.; Moore, J.; Meng, J.; Zhang, Y.; Cheng, Z. and Yu, I. (2007): Inhibitory effect of Chardonnay and black raspberry seed extracts on lipid oxidation in fish oil and their radical scavenging and antimicrobial properties. Food Chem. 104: 1065-1073.

Megahed, A.A. and Hafez, A.A. (2012): Antimicrobial effect of cumin, dill and chili aqueous extracts on growth of some pathogens in white soft cheese. Egyptian Journal of Food Safety, 1: 40-45.

Menon, V.P. and Sudheer, A.R. (2007): Antioxidant and antinflammatory properties of Curcumin. Adv. Eep. Med. Biol. 595: 105-125.

Murad, H.A. (1998): Antimicrobial activity of some natural extracts and its potentiality for controlling yeast growth in yoghurt. J. Agric. Sci. Mansoura Univ. 23: 6115-6125.

Omer, I.A. (2014): Effect of cumin oil concentrations on chemical composition and sensory characteristics of Sudanese white cheese during ripening. Int. J. Curr. Microbiol. App. Sci. 3: 961-968.

Punithavathi, D.; Venkatesan, N. and Babu, M. (2000): Curcumin inhibition of bleomycinInduced pulmonary fibrosis in rats. Br. J. Pharmacol. 131: 169-172.

Samir, A.M.; Mohamed, F.R. and Kahlid, M.E. (2013): Cold pressed Nigella Sativa oil inhibits the growth of foodborne pathogens and improve quality of Domiati cheese. journal of Food Safety, 33: 470-480.

SAS Institute. (2009): Base SAS 9.2 procedures guide. Cary, NC: SAS Institute, Inc. Retrieved March 10, 2010, from the SAS product Documentation web.

Siddiqui, A.M.; Cui, X.; Dong, R.W.; Zhou, M.; Simms, H. and Wang, P. (2006): The antiinflammatory effect of Curcumin in an experimental model of sepsis is mediated by up-regulation of peroxisome proliferatoractivated receptor-gamma. Cirt. Care. Med. 34: 1874-1882.

Sumner, S.S.; Roche, F. and Taylor, S.I. (1990): Factor controlling histamine production in Swiss cheese inoculated with Lactobacillus buchneri. J. Dairy Sci., 73: 3050-3058.

Suzuki, M.; Nakamura, T.; Lyoki, S.; Fujiwara, A.; Watanabe, Y.; Mohri, K.; Isobe, K.; Ono, K. and Yano, S. (2005): Elucidation of antiallergic activities of Curcumin-related 
compounds with a special reference to their anti-oxidative activities. Biol. Pharm. Bull. 28: 1438-1443.

Tajbakash, S.; Mohammadi, K.; Deilami, I.Z.; Zandi, K.; Fouladvand, M.; Ramedani, E. and Asayash, G. (2008): Antibacterial activity of indium Curcumin and inium diacetylcurcumin. Afr. J. Biote.7: 3832-3835.

Thongson, C.; Davidson, PM.; Mahakarnchanakul, W. Vibulsresth, P. (2005): Antimicrobial effect of Thai spices against Listeria monocytogenes and Salmonella typhimurium DT104. J Food Prot. 68: 2054-2058.
Wahba, N.M.; Ali, M.M. and Ahmed, E.K. (2004): Effect of Nigella sativa and salt on the growth and survival of E. coli O157: $\mathrm{H} 7$ during manufacture and storage of Damietta cheese. Assiut Vet Med J. 50: 74-83.

World Health Organization (WHO) (2002): Food safety and food borne illness. World Health Organization, Geneva, pp 1-4.

Yousef, H.M. Sobieh and Nagedan, K. (2001): Microbial status of Domiati cheese at $\mathrm{Al}$ Gassiem area, Saudi Arabia.. $8^{\text {th }}$ Sci. Cong., The Fac. Vet. Med. Assuit University, pp: 91-97.

\section{تحسيين جودة الجبن الامياطي باستخدام مستخلصات بعض البهارات \\ جيهان اسعاعيل ابر/هيم ، امام حسين عبا الحكيم}

Email: emamhu@yahoo.com. Assiut University web-site: www.aun.edu.eg

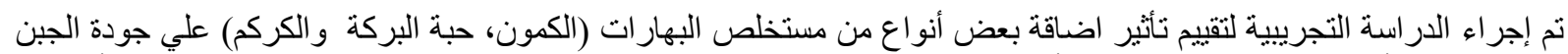

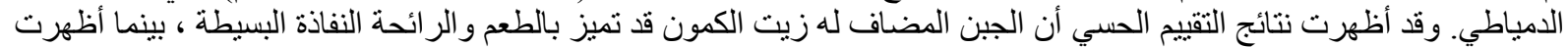

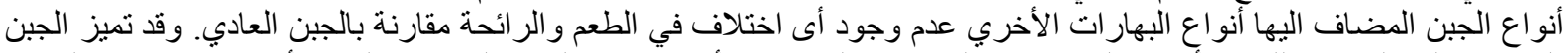

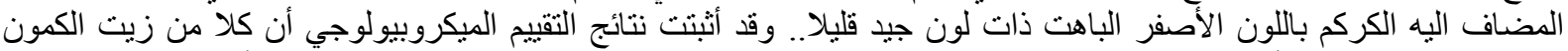

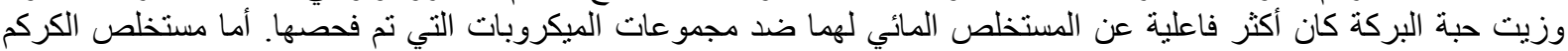

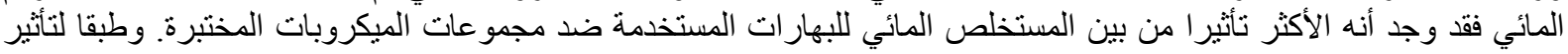

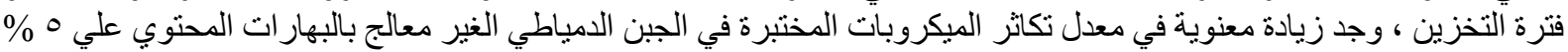

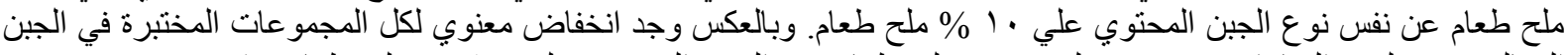

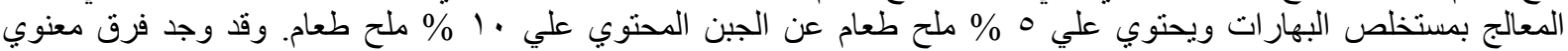

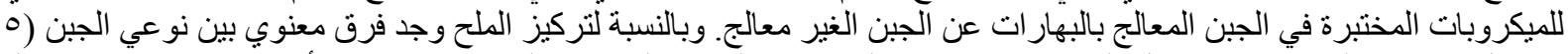

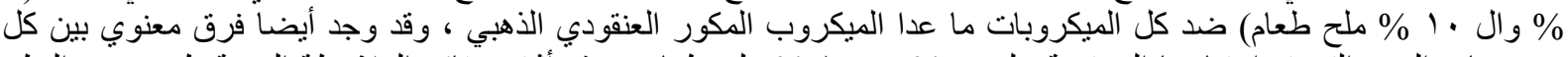

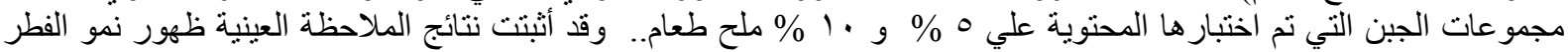
و الخمائر علي سطح الجبن الغير معالج بالبهار ات (0 \% ملح طعام) و الثرش الخاص به فقط عند ام آ يوم من بدء التجربة. 